\title{
ISOSPIN MASS SPLITTINGS OF BARYONS IN POTENTIAL MODELS
}

\author{
Kálmán Varga \\ Institute for Nuclear Research of the Hungarian Academy of Sciences \\ Debrecen, PO Box 51, Hungary \\ and RIKEN, Hirosawa 2-1, Wako, Saitama 35101, Japan \\ Marco Genovese*, Jean-Marc Richard and Bernard Silvestre-Brac \\ Institut des Sciences Nucléaires \\ Université Joseph Fourier-CNRS-IN2P3 \\ 53, avenue des Martyrs, F-38026 Grenoble
}

(May 14, 2021)

\begin{abstract}
We discuss the isospin-breaking mass differences among baryons, with particular attention in the charm sector to the $\Sigma_{c}^{+}-\Sigma_{c}^{0}, \Sigma_{c}^{++}-\Sigma_{c}^{0}$, and $\Xi_{c}^{+}-\Xi_{c}^{0}$ splittings. Simple potential models cannot accommodate the trend of the available data on charmed baryons. More precise measurements would offer the possibility of testing how well potential models describe the nonperturbative limit of QCD.

14.20.-c, 14.20.Gk, 14.20.Lq, 14.20.Jn
\end{abstract}

*Supported by the EU Program ERBFMBICT 950427 


\section{INTRODUCTION}

A successful phenomenology of the hadron spectrum has been obtained using nonrelativistic potential models, which tentatively simulate the low-energy limit of QCD [1 3 ]. The interquark potential usually contains a linear part which describes QCD confinement and is supplemented by a Coulomb term which may be attributed to one-gluon exchange. Spin-spin, spin-orbit and tensor terms are added, analogous to the Fermi-Breit components of QED potential, derived from $v / c$ expansion.

There are obvious difficulties. Large relativistic corrections can be anticipated for light quarks. The naive superposition of a Coulomb and a linear term may be too schematic.

Results rely on the potential at intermediate distances $(0.1 \mathrm{fm} \lesssim r \lesssim 0.5 \mathrm{fm})$ where neither the perturbative nor the string limit holds. Nevertheless, the success of potential models indicates that somehow delicate relativistic and field-theory effects are hidden in the parameters. As none of the more ambitious approaches, for instance lattice calculation [4], is yet able to produce very precise results, it is still justified to use potential models as tools to analyze hadron properties, with the hope of better understanding the non-perturbative limit of QCD.

Among the observables of interest, isospin-violating mass differences have retained much attention. Earlier studies of these mass differences [5] have been reconsidered within constituent-quark models. In general, the $n-p, \Sigma^{-}-\Sigma^{0}, \Sigma^{-}-\Sigma^{+}, \Xi^{-}-\Xi^{0}$ splittings of the nucleon, $\Sigma$ and $\Xi$ multiplets are well reproduced, this fixing the quark-mass difference $\Delta m=m_{d}-m_{u}$. Predictions for charmed baryons can then be supplied. Some results

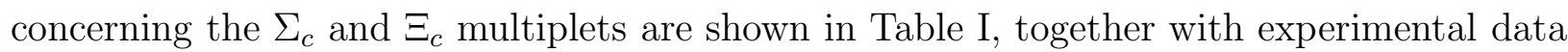
[6]. The estimates of Wright [7] and of Deshpande et al. [8] are not really potential models, they are shown only for information and comparison with the others 9 17

Some of the models include only a fraction of the possible contributions, for instance 
electrostatic interaction is accounted for, but the mass dependence of the chromomagnetic interaction is neglected when replacing a $d$ quark by a $u$ one. This is hardly justified. As underlined, e.g., by Isgur [16], these isospin splittings arise from several canceling contributions, so that each effect should be carefully computed and even small terms should be incorporated.

The most striking feature of Table $\mathbb{Q}$ is the wide spread of predictions. Next come the observation that none of the models is compatible with the presently available data [6]. In particular the predicted $\Xi_{c}^{0}-\Xi_{c}^{+}$splitting tends to be smaller than the PDG average $6.3 \pm 2.1$ $\mathrm{MeV}$ [6]. However, a preliminary result by CLEO indicates a smaller value $\Xi_{c}^{0}-\Xi_{c}^{+}=$ $2.5 \pm 1.7 \pm 1.1 \mathrm{MeV}[18]$. This collaboration has also detected candidates for the internal spin excitations $\Xi_{c}^{\prime 0}$ and $\Xi_{c}^{\prime+}$ (total spin $1 / 2$ with the light-quark pair $s d$ or $s u$ mostly in a spin-triplet state). Their measurements indicate a splitting $\Xi_{c}^{\prime 0}-\Xi_{c}^{\prime+} \simeq 1.7 \mathrm{MeV}$ with a large error bar. There are also data from CLEO on the $\Xi_{c}^{*}$ states 18 .

The $\Sigma_{c}$ multiplet is the most puzzling. The $\Sigma_{c}^{++}-\Sigma_{c}^{0}$ splitting is usually larger than $\Sigma_{c}^{+}-\Sigma_{c}^{0}$, while data seemingly favour the reverse. In other words, most models predict an ordering of $\Sigma_{c}^{++}, \Sigma_{c}^{+}$and $\Sigma_{c}^{0}$ which is not seen, to the extent one can draw any conclusion from the data.

The present investigation is motivated by the discrepancy between data and models. We wish to understand whether this problem points out a general limitation of potential models, in particular those based on one gluon-exchange, or can be solved by reconsidering the choice of parameters and removing unjustified approximations in the three-body problem.

For this purpose, first we carefully estimate the role of each contribution to the splittings within specific potential models. This should measure to which extent previous calculations suffer from neglecting some effects or treating them approximately. Then we analyse the sensitivity to the choice of potential, to see whether fitting the data can be achieved by an appropriate tuning of parameters or it is out of reach of this approach. Predictions are 
listed for a number of isospin multiplets, making possible a comparison with all available experimental data, and with those one could expect to be measured in the near future.

\section{MODEL CALCULATION}

A representative quark model is proposed in Ref. [2], where the potential is tentatively designed to fit both meson and baryon spectra, using the empirical rule

$$
V_{Q Q Q}\left(\overrightarrow{\mathrm{r}}_{1}, \overrightarrow{\mathrm{r}}_{2}, \overrightarrow{\mathrm{r}}_{3}\right)=\frac{1}{2} \sum_{i<j} V\left(\left|\overrightarrow{\mathrm{r}}_{i}-\overrightarrow{\mathrm{r}}_{j}\right|\right) .
$$

In [2], the quark-antiquark potential reads

$$
V(r)=-\frac{\kappa}{r}+\lambda r^{p}-\Lambda+\frac{2 \pi \kappa^{\prime}}{3 m_{1} m_{2}} \tilde{\delta}\left(r, r_{0}\right) \vec{\sigma}_{1} \cdot \vec{\sigma}_{2}
$$

with $\tilde{\delta}\left(r, r_{0}\right)=\exp \left(-r / r_{0}\right) /\left(4 \pi r_{0}^{2} r\right)$ being a smeared form of the contact term. The parameters are (in the units used by the authors [2]):

$$
\begin{gathered}
p=1, \quad \kappa=102.67 \mathrm{MeV} . \mathrm{fm}, \quad \kappa^{\prime}=6 \times 102.67 \mathrm{MeV} . \mathrm{fm}, \\
(\mathrm{BCN}) \quad \lambda=1 /(0.0326)^{2} \mathrm{MeV} / \mathrm{fm}, \quad \Lambda=913.5 \mathrm{MeV}, \quad r_{0}^{-1}=2.2 \mathrm{fm}^{-1}, \\
m_{q}=337, \quad m_{s}=600, \quad m_{c}=1870, \quad m_{b}=5259 \mathrm{MeV} .
\end{gathered}
$$

One of the difficulties in the above model is that the spatial extension $r_{0}$ of the spinspin term is too large to describe a short-range interaction between heavy quarks. As a consequence, the $J / \Psi-\eta_{c}$ hyperfine splitting is not well reproduced. This is why Ref. [3], following for instance Ref. [19], introduces a flavour dependence in $r_{0}$, namely

$$
r_{0}\left(m_{i}, m_{j}\right)=A\left(\frac{2 m_{i} m_{j}}{m_{i}+m_{j}}\right)^{-B}
$$

while a Gaussian form $\tilde{\delta}\left(r, r_{0}\right)=\exp \left(-r^{2} / r_{0}^{2}\right) /\left(\pi^{3 / 2} r_{0}^{3}\right)$ is now adopted. Both models AL1 and AP1 of Ref. [3] fit very well the meson spectrum. The parameters are 


$$
\begin{aligned}
p & =1, \quad \kappa=0.5069, \quad \kappa^{\prime}=1.8609 \quad \lambda=0.1653 \mathrm{GeV}^{2}, \\
\text { (AL1) } \quad \Lambda & =0.8321 \mathrm{GeV}, \quad B=0.2204, \quad A=1.6553 \mathrm{GeV}^{B-1}, \\
m_{q} & =0.315, \quad m_{s}=0.577, \quad m_{c}=1.836, \quad m_{b}=5.227 \mathrm{GeV},
\end{aligned}
$$

and

$$
\begin{gathered}
\quad p=2 / 3, \quad \kappa=0.4242, \quad \kappa^{\prime}=1.8025, \quad \lambda=0.3898 \mathrm{GeV}^{5 / 3} \\
(\mathrm{AP} 1) \quad \Lambda=1.1313 \mathrm{GeV}, \quad B=0.3263, \quad A=1.5296 \mathrm{GeV}^{B-1}, \\
m_{q}=0.277, \quad m_{s}=0.553, \quad m_{c}=1.819, \quad m_{b}=5.206 \mathrm{GeV} .
\end{gathered}
$$

None of these models include tensor forces, since this interaction is not expected to give important contributions in hadron spectroscopy, at least for ground states [20].

For studying isospin breaking, we have allowed for $m_{u} \neq m_{d}$ and added to the potential the electrostatic interaction between quarks. The difference $\Delta m=m_{d}-m_{u}$ between $d$ and $u$ quark masses has been adjusted to reproduce neutron-proton and $\Sigma^{-}-\Sigma^{+}$mass splittings. In particular, we have taken $m_{u}=327 \mathrm{MeV}$ and $m_{d}=338 \mathrm{MeV}$ for AL1, $m_{u}=337 \mathrm{MeV}$ and $m_{d}=353.85 \mathrm{MeV}$ for BCN and $m_{u}=277 \mathrm{MeV}$ and $m_{d}=300.5 \mathrm{MeV}$ for AP1. Our estimate of $\Delta m$ for constituent quarks is larger than the common wisdom for current quarks, $\Delta m \simeq 4 \mathrm{MeV}$. However, it must be noticed that dressing quarks modifies this quantity, for the cloud of virtual states depends on the flavour of the quark it is surrounding [21,22].

Baryon masses have been obtained using two reliable numerical methods [23,24]. In the first case every contribution was included non-perturbatively in the variational procedure, while in the second one the electromagnetic terms were treated perturbatively. The perfect agreement of two results indicates that a good convergence has been reached.

The various contributions to $n-p$ and to charmed baryons mass differences are shown

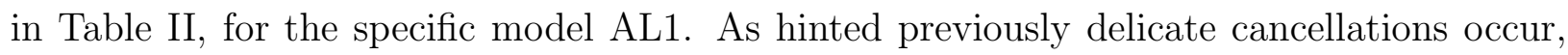
requiring an accurate treatment of each term. In a flavour-independent potential the energy 
of a given state decreases when any of the constituent masses is increased, i.e. $(\Delta T+\Delta W+$ $\Delta B) / \Delta m<0$ in the notations of Table II. This is observed in our calculations, though in the $\Xi_{c}$ case, the flavour dependence of spin-spin term goes in the opposite direction. As for the kinetic energy itself, one expects [26] $\Delta T / \Delta m>0$ if confinement dominates the binding process, and $\Delta T / \Delta m<0$ when the Coulomb part becomes more influential.

Before discussing the results obtained for baryons, we have investigated the splittings among mesons, for instance $D^{+}(c \bar{d})-D^{0}(c \bar{u})$. The results are shown in Table $\mathbb{I I}$, for the three models BCN, AL1 and AP1. $\Delta m$ is adopted to reproduce $m_{n}-m_{p}$ in the baryon sector. An acceptable agreement is found for AL1, while BCN and AP1 seem to be disfavoured, leading to a larger overestimation of the $K^{0}-K^{+}$splitting. Actually, AL1 is somehow an improvement of BCN, while the confining part $\propto r^{2 / 3}$ of $\mathrm{AP} 1$ does not agree with lattice results and fails for heavy mesons as well. In the following AL1 will be our benchmark, the results for $\mathrm{AP} 1$ and $\mathrm{BCN}$ will also be given in order to show how stable are the predictions respect to the choice of the potential.

Our results for baryons splittings are shown in Table एV.

A check of the consistency of our calculation is provided by the relations listed by Franklin [25]. When one switches off the electromagnetic interaction, one deals with energies which evolve continuously when going, for instance, from $(Q u u)$ to $(Q d d)$ via $(Q u d)$. Hence a mass combination

$$
\delta\left(\Sigma_{Q}\right)=(Q u u)+(Q d d)-2(Q u d)
$$

receives contributions mostly from Coulomb effects. If the latter are treated at first order with a wave function $(Q q q)$ properly averaging that of $(Q u u),(Q d d)$ and $(Q d u)$, then $\delta\left(\Sigma_{Q}\right) \simeq \alpha\left\langle r_{q q}^{-1}\right\rangle$, i.e. the charge of heavy quark $Q$ disappears.

If furthermore the $(q q)$ part of the of the $(Q q q)$ wave function does not depend much on the mass of the heavy quark $Q$ and on the coupling of the spin of $Q$ to the $(q q)$ spin triplet, 
then $\delta\left(\Sigma_{Q}\right)$ should be approximately the same for $\Sigma, \Sigma^{*}, \Sigma_{c}$ or $\Sigma_{b}$ multiplets. This is again rather well verified in our calculation.

The possible dependence of the $(q q)$ distribution on the mass of the third quark $Q$ is discussed by Rosner [26] as a "three-body effect". It was investigated previously [27,28] in the literature, not for $\left\langle r_{q q}^{-1}\right\rangle$, but for the somewhat similar matrix element $\left\langle\delta^{(3)}\left(\overrightarrow{\mathrm{r}}_{q q}\right)\right\rangle$ that enters the calculation of the hyperfine splittings in usual quark models. In an approach à la Breit-Fermi, the ratio

$$
R=\frac{2 \Sigma^{*}+\Sigma-3 \Lambda}{2 \Delta-2 N}
$$

reveals the ratio of the $(q q)$ short-range correlations in $(s q q)$ and $(q q q)$. Similarly the ratio

$$
R^{\prime}=\frac{\Xi^{*}-\Xi}{\Sigma^{*}-\Sigma}
$$

gives a comparison of $(q s)$ correlations in $(s s q)$ and $(s q q)$. The experimental values $R \simeq$ 1.04 and $R^{\prime} \simeq 1.12$, as well as the detailed three-body calculations [27,28] show that, as conjectured by Rosner [26], the $q_{1}$ and $q_{2}$ quarks tend to bind more intimately within $\left(q_{1} q_{2} q_{3}\right)$ when $q_{3}$ becomes heavier. The effect is about 5 to $10 \%$ when $q_{3}$ changes from ordinary to strange, may be slightly more from strange to charmed. In would be desirable to reach a deeper understanding of this property, beyond numerical investigations. A possible starting point is given by the harmonic oscillator

$$
H=p_{1}^{2}+p_{2}^{2}+\alpha p_{3}^{2}+r_{12}^{2}+\beta\left(r_{13}^{2}+r_{23}^{2}\right)
$$

where the $r_{12}$ dependence of the wave function factorizes out and is easily shown to be independent of the inverse mass $\alpha$ (but it does depend on the strength $\beta$ ). 


\section{DISCUSSION}

The isospin-violating splittings of light and heavy baryons are shown in Table [V]. The $\Sigma^{-}-\Sigma^{0}$ and $\Delta^{0}-\Delta^{++}$splittings and even (within large errors) the electromagnetic splittings for the excited states $\Sigma^{*}(1385)$ and $\Xi^{*}(1530)$ come out in good agreement with the experimental data [6]. Some problems appear however for charmed baryons.

More precisely, while the experimental datum $\Sigma_{c}^{++}-\Sigma_{c}^{0}=0.8 \pm 0.4 \mathrm{MeV}$ is well reproduced, one finds an, albeit small (of the order of $-0.5 \mathrm{MeV}$ ), negative $\Sigma_{c}^{+}-\Sigma_{c}^{0}$, at variance with the experimental datum $\Sigma_{c}^{+}-\Sigma_{c}^{0}=1.4 \pm 0.6 \mathrm{MeV}$. Also the result for $\Xi_{c}^{+}-\Xi_{c}^{0}=2.2$ $\mathrm{MeV}$ is smaller than the, still rather imprecise, experimental datum $\Xi_{c}^{+}-\Xi_{c}^{0}=6.3 \pm 2.3$ or $4.7 \pm 2.1 \mathrm{MeV}$, where the first number corresponds to the particle data group average and the second to their fit. The problem is not solved using AP1 or BCN.

To summarize at this stage, the splittings of charmed baryons do not agree with experimental results, when they are calculated from potential models supplemented by electrostatic forces and a mass difference $\Delta m$ between $d$ and $u$ quarks.

Reasonable changes of light quark masses do not modify substantially this situation.

An effect which we have neglected up to now is the electromagnetic dipole-dipole interaction between quarks, whose dominant term (neglecting the small contribution of components of the wave function with non-vanishing angular momentum) is:

$$
-\frac{2 \pi}{3} \frac{q_{i} q_{j} \alpha}{m_{i} m_{j}} \delta\left(r_{i j}\right) \vec{\sigma}_{i} \cdot \vec{\sigma}_{j}
$$

where $q_{i}$ are quark charges in units of electron charge. Albeit surely present, one expects it to be smaller than usual Coulomb interaction. Our numerical results including this dipoledipole term are shown in IV. We used a regularized form $\tilde{\delta}$ for $\delta(r)$, taken to be the same as for the strong spin-spin force. The magnetic contribution goes in the right direction, 
but remains too small to push the computed masses significantly closer to the experimental ones.

This problem raises the question whether some contribution has been forgotten. For example, in some models adjusted to reproduce meson and baryon masses simultaneously introduce in the baryon sector an ad-hoc 3-body term of the form [2.3]

$$
D_{3}+\frac{A_{3}}{\left(m_{1} m_{2} m_{3}\right)^{b_{3}}} .
$$

This parameterization is purely empirical. For the AL1 model, the parameters are $D_{3}=$ $0.07376, A_{3}=-0.05546$ and $b_{3}=1 / 4$.

As it depends on masses, this term gives a contribution to isospin breaking effects as well. The 3-body term (12) slightly improves the description of the electromagnetic splittings of light baryons. However, how is evident by inspecting Eq. (12), the contribution $\Sigma_{c}^{+}-\Sigma_{c}^{0}$ goes in the wrong direction. When the 3-body term (12) is accounted for one obtains typically $\Sigma_{c}^{+}-\Sigma_{c}^{0} \simeq-0.7 \mathrm{MeV}$ (instead of $-0.55 \mathrm{MeV}$ ), and $\Xi_{c}^{0}-\Xi_{c}^{+} \simeq 1.5 \mathrm{MeV}$ (instead of $2.58 \mathrm{MeV}$ ), with little dependence on the choice of parameters. Of course, one could think of more complicated three-body interactions, but their form remains completely arbitrary and somehow the appealing features of potential models are lost once one violates flavour independence and gives up the link between quark-quark and quark-antiquark forces.

Another possibility which can be explored is the running of $\alpha_{s}$, which leads to a reduced coupling when heavy quarks appears for the scale is chosen to be proportional to the masses involved (of course problems related to the precise choice of the scale and to the unknown $\alpha_{s}$ behaviour at small scales emerge). Such an effect would decrease the strenght of the spinspin term involving heavy quarks, but this would not go in the right direction for changing the order of $\Sigma_{c}$ states.

Finally, we discuss now two interaction terms which have been contemplated in addition or in replacement of chromomagnetism. 
The first comes from the contribution of instantons. The non-relativistic form of a potential mimicking 't Hooft interaction [29] has been elaborated in Ref.s [30,31]. The value of the coupling must however be fixed phenomenologically. Interesting results have been obtained on hadron spectroscopy with models including this instanton term replacing [30,31] or supplementing [32] the chromomagnetic force.

However, when the instantonic potential is considered only as a further correction to Eq. (2), it does not contribute substantially to $\Sigma_{c}$ mass splittings, for it is inversely proportional to the quark masses and vanishes for a quark pair with spin 1. Thus it cannot help solving the problem of $\Sigma_{c}$ splittings. It gives, anyway, a positive contribution, albeit not to be expected quite large (of course the precise numerical value will depend on the choice of the coupling), to $\Xi_{c}^{+}-\Xi_{c}^{0}$.

The second type of interaction deals with meson exchange between quarks. There is a rich literature on the subject, which has recently be revisited by Glozman and collaborators (see e.g. Refs. [33] and references therein), who have adopted rather an extreme point of view where the chromomagnetic force is completely removed. These authors obtain a surprisingly good fit to light and strange baryons. In this approach, the study of electromagnetic splittings of baryons is somewhat reminiscent of isospin violating effects in nuclear physics, where one accounts for a difference between $\pi^{ \pm}$and $\pi^{0}$ masses and their couplings to nucleons. This remains to be studied. However, the extension of Glozman model to heavy baryons seems problematic, notwithstanding some initial attempts [35.

\section{OUTLOOK}

In conclusion, we find that non-relativistic potential models do not permit to reproduce the data on $\Sigma_{c}^{+}-\Sigma_{c}^{0}$ and $\Xi_{c}^{+}-\Xi_{c}^{0}$ mass splittings, despite the good agreement obtained for light baryons. Of course experimental data need further confirmation, and need to be 
extended to beauty and double-charm sectors, to see if this discrepancy persists.

Previously, Franklin [25] pointed out that the experimental data on charmed baryons violate mass relations which are expected to hold within large class of quark models. We have checked that the mass obtained from an accurate solution of the three-body problem fulfill the Franklin relations. So if the difficulty persists, its solution should be searched in a intrinsic limitation of usual quark models, for instance in the need for new dynamical contributions, such as electromagnetic penguins [36].

The present situation is somewhat a paradox. The $\Lambda_{c}$ and $\Omega_{c}$ and the average $\Sigma_{c}$, $\Xi_{c}$ states are reasonably described by simple potentials, i.e., one seemingly controls the behaviour of the ground-state baryons when an ordinary quark is replaced by a charm one. Meanwhile one does not understand the effect of a more modest move, when a up quark is changed in a down one.

\section{Acknowledgements}

Stimulating discussions with Fl. Stancu and S. Pepin are gratefully acknowledged, as well as correspondence from S.C. Timm, J. Franklin and T. Goldman.

[1] See, for example, R. K. Bhaduri, "Models of the Nucleon", ed. Addison-Wesley Publ. (1988) and refs. therein.

[2] R. K. Bhaduri et al., Nuovo Cimento A 65 (1981) 376.

[3] See B. Silvestre-Brac, Few-body Systems 20 (1996) 1, and refs. therein.

[4] See Proc. of Lattice 96, St. Louis (USA) 4-8 June 1996, Nucl. Phys. B (Proc. Suppl.) 53 (1997). 
[5] Y. Miyamoto, Progr. Th. Phys. 35 (1966) 179.

[6] Review of Particle Properties, R. M. Barnett et al., Phys. Rev. D 54 (1996).

[7] A. C. Wright, Phys. Rev. D 17 (1978) 3130.

[8] N. Deshpande et al., Phys. Rev. D 15 (1977) 1885.

[9] C. Itoh et al., Progr. Th. Phys. 54 (1975) 908.

[10] S. Ono, Phys. Rev. D 15 (1977) 3492.

[11] L. Chan, Phys. Rev. D 15 (1977) 2478.

[12] K. Lane and S. Weinberg, Phys. Rev. Lett. 37 (1976) 717.

[13] D.B. Lichtenberg, Phys. Rev. D 16 (1977) 231.

[14] C.S. Kalman and G. Jakimow, Lett. al Nuovo Cimento 19 (1977) 403.

[15] S. Capstick, Phys. Rev. D 36 (1987) 2800.

[16] N. Isgur, Phys. Rev. D 21 (1980) 779.

[17] J.-M. Richard and P. Taxil, Z. Phys. C 26 (1984) 421.

[18] CLEO collaboration, preprint CLEO CONF 97-29 (available at http://www.lns.cornell.edu/public/CONF/1997/CONF97-29/conf_97-29.ps);

Steven C. Timm, Contribution at "Hadron97", Int. Conf. hadron Spectroscopy, Proc. to be edited by S.U. Chung at A.I.P.

[19] S.Ono and F. Schöberl, Phys. Lett. B 118 (1982) 419.

[20] See, e.g., N. Isgur, G. Karl and R. Koniuk, Phys. Rev. D 25 (1982) 2394; Phys. Rev. Lett. 41 (1978) 1269 .

[21] R. P. Feynman and R. D. Field, Phys. Rev. D 15 (1977) 2590.

[22] See, e.g., R. Ball et al., Phys. Lett. B 329 (1994) 505 and ref.s therein. 
[23] K. Varga and Y. Suzuki, Phys. Lett. B 396 (1997) 1, Phys. Rev. C 52 (1995) 2885.

[24] B. Silvestre-Brac and C. Gignoux, Phys. Rev. D 32 (1985) 743.

[25] J. Franklin, Phys. Rev. D 53 (1996) 564.

[26] J. L. Rosner, preprint hep-ph/9707473.

[27] I. Cohen and H. I. Lipkin, Phys. Lett. B 106 (1981) 119.

[28] J.-M. Richard and P. Taxil, Ann. Phys. (N.Y.) 150 (1983) 267.

[29] G. 't Hooft, Phys. Rev. D 14 (1976) 3432.

[30] A. E. Dorokhov and N. I. Kochelev, JINR preprint Dubna E-2-86-224 (1986), Sov. J. Nucl. Phys. 52 (1990) 135, A. E. Dorokhov Nucl. Phys. A 581 (1995) 654.

[31] See, also, W. H. Blask et al., Z. Phys. A 337 (1990) 327.

[32] C. Semay and B. Silvestre-Brac, Nucl. Phys. A 618 (1997) 455.

[33] L.Y. Glozman and D.O. Riska, Phys. Rep. 268 (1996) 263, L.Y. Glozman et al., preprint UNIGRAZ-UTP 26-06-97.

[34] A. E. Dorokhov and N. I. Kochelev, Z. Phys. C 37 (1988) 377.

[35] L.Y. Glozman and D. O. Riska, Nucl. Phys. A 603 (1996) 326.

[36] G.J. Stephenson, K. Maltman and T. Goldman, Phys. Rev. D 43 (1991) 860; L.S. Kisslinger, T. Goldman and Z. Li, Phys. Lett. B 416 (1998) 263.

\section{List of Tables}

I Predictions of different models for charmed baryons electromagnetic mass splitting . . . . . . . . . . . . . . . . . . . . . . . . . 14 
II Different contributions (in $\mathrm{MeV}$ ) to the neutron to proton and charmed baryons mass differences: $\Delta m$ is the change of constituent quark masses, $\Delta T$ the difference of kinetic energies, $\Delta W$ the variation of the expectation value of the Wigner term (independent on spin and isospin), $\Delta B$ the difference of Bartlett components $\left(\propto \bar{\sigma}_{i} \bar{\sigma}_{j}\right)$ and finally $\Delta C$ comes from the Coulomb electric interaction. Here we use the model AL1. . . . . . . . . . . . . . . . 15

III Comparison of the isospin-breaking splittings of mesons (in MeV) obtained from several potential models: Bhaduri et al. (BCN); Silvestre-Brac and Semay (AL1 with linear confinement, AP1 with a $r^{2 / 3}$ confinement). . . . . . . 16

IV Comparison of the isospin-breaking splittings (in $\mathrm{MeV}$ ) obtained from sev eral potential models: Bhaduri et al. (BCN); Silvestre-Brac and Semay (AL1 with linear confinement, AP1 with a $r^{2 / 3}$ confinement); Richard and Taxil where the hyperfine interaction is treated perturbatively (RT II has a linear central potential, RT I a $r^{0.1}$ one). In the column AL1 + dd, the magnetid dipole-dipole interaction between quarks is accounted for, in addition to the electrostatic potential. Some redundant splittings are shown for the ease of discussion . . . . . . . . . . . . . . . . . . . . 17

TABLE I. Predictions of different models for charmed baryons electromagnetic mass splittings

\begin{tabular}{lccc}
\hline \hline Model & $\Sigma_{c}^{++}-\Sigma_{c}^{0}$ & $\Sigma_{c}^{+}-\Sigma_{c}^{0}$ & $\Xi_{c}^{0}-\Xi_{c}^{+}$ \\
\hline Experiment [6] & $0.8 \pm 0.4 \mathrm{MeV}$ & $1.4 \pm 0.6 \mathrm{MeV}$ & $6.3 \pm 2.1 \mathrm{MeV}$ \\
Wright [7] & -1.4 & -2.0 & 3.1 \\
Deshpande et al. [8] & $-(3-18)$ & $-(2.5-10)$ & $4.5-12$ \\
Itoh [9] & 6.5 & 2.4 & 2.5 \\
Ono [10] & 6.1 & 2.2 & 1.8 \\
Lane and Weinberg [12] & -6 & -4 & 4 \\
Chan [1] & 0.4 & -0.7 & 3.2
\end{tabular}




\begin{tabular}{|c|c|c|c|}
\hline Lichtenberg [13] & 3.4 & 0.8 & 1.1 \\
\hline Kalman and Jakimow 14 & -2.7 & -2.2 & 3.6 \\
\hline Capstick 15 & 1.4 & -0.2 & \\
\hline Isgur 16 & -2 & -1.8 & \\
\hline \multirow{2}{*}{ Richard and Taxil [17] } & 3 & 1 & 0 \\
\hline & -2 & -1 & 2 \\
\hline
\end{tabular}

TABLE II. Different contributions (in $\mathrm{MeV}$ ) to the neutron to proton and charmed baryons mass differences: $\Delta m$ is the change of constituent quark masses, $\Delta T$ the difference of kinetic energies, $\Delta W$ the variation of the expectation value of the Wigner term (independent on spin and isospin), $\Delta B$ the difference of Bartlett components $\left(\propto \overrightarrow{\sigma_{i}} \overrightarrow{\sigma_{j}}\right)$ and finally $\Delta C$ comes from the Coulomb electric interaction. Here we use the model AL1.

\begin{tabular}{ccccccc}
\hline \hline Baryons & $\Delta m$ & $\Delta T$ & $\Delta W$ & $\Delta B$ & $\Delta C$ & Total \\
$n-p$ & 11 & 0.33 & -6.45 & -2.86 & -0.76 & 1.24 \\
$\Sigma_{c}^{+}-\Sigma_{c}^{0}$ & -11 & 0.56 & 7.55 & 1.39 & 1.14 & -0.35 \\
$\Sigma_{c}^{++}-\Sigma_{c}^{0}$ & -22 & 0.79 & 15.78 & 2.07 & 4.53 & 1.20 \\
$\Xi_{c}^{0}-\Xi_{c}^{+}$ & 11 & -3.31 & -5.56 & 1.72 & -1.01 & 2.83 \\
\hline \hline
\end{tabular}


TABLE III. Comparison of the isospin-breaking splittings of mesons (in MeV) obtained from several potential models: Bhaduri et al. (BCN); Silvestre-Brac and Semay (AL1 with linear confinement, AP1 with a $r^{2 / 3}$ confinement).

\begin{tabular}{ccccc}
\hline \hline Splitting & Exp. (Ref. [6]) & BCN & AL1 & AP1 \\
\hline$K^{0}-K^{+}$ & $3.995 \pm 0.034$ & 13.15 & 6.64 & 9.56 \\
$K^{* 0}-K^{*+}$ & $6.7 \pm 1.2$ & 1.55 & 1.36 & 1.28 \\
$D^{+}-D^{0}$ & $4.78 \pm 0.10$ & 5.37 & 3.78 & -0.33 \\
$D^{*+}-D^{* 0}$ & $2.6 \pm 1.8$ & 2.44 & 2.74 & -0.16 \\
$B^{0}-B^{-}$ & $0.35 \pm 0.29$ 口 & -1.46 & -1.29 & -6.06 \\
$B^{* 0}-B^{*-}$ & & -2.04 & -1.23 & -5.26 \\
\hline \hline
\end{tabular}

\footnotetext{
${ }^{1}$ Notice that on four available measurements, two are negative [6]
} 
TABLE IV. Comparison of the isospin-breaking splittings (in MeV) obtained from several potential models: Bhaduri et al. (BCN); Silvestre-Brac and Semay (AL1 with linear confinement, AP1 with a $r^{2 / 3}$ confinement); Richard and Taxil where the hyperfine interaction is treated perturbatively (RT II has a linear central potential, RT I a $r^{0.1}$ one). In the column AL1 + dd, the magnetic dipole-dipole interaction between quarks is accounted for, in addition to the electrostatic potential. Some redundant splittings are shown for the ease of discussion.

\begin{tabular}{|c|c|c|c|c|c|c|c|}
\hline Splitting & Exp. [Ref] & $\mathrm{BCN}$ & AL1 & AP1 & RT II & RT I & $\mathrm{AL} 1+\mathrm{dd}$ \\
\hline$n-p$ & $\begin{array}{c}1.293318 \pm \\
0.0000009 \text { [6] }\end{array}$ & 1.38 & 1.16 & 1.29 & 1.2 & 1.3 & 1.24 \\
\hline$\Delta^{+}-\Delta^{++}$ & & 0.54 & 0.08 & 2.08 & & & 0.36 \\
\hline$\Delta^{0}-\Delta^{++}$ & $2.7 \pm 0.3$ & 3.21 & 2.20 & 6.10 & & & 2.54 \\
\hline$\Delta^{-}-\Delta^{++}$ & & 8.04 & 6.34 & 11.38 & & & 6.55 \\
\hline$\Sigma^{-}-\Sigma^{0}$ & $4.88 \pm 0.08$ [6] & 7.09 & 5.16 & 6.07 & 2 & 4 & 5.24 \\
\hline$\Sigma^{-}-\Sigma^{+}$ & $8.09 \pm 0.16$ [6] & 11.98 & 8.25 & 10.57 & 4 & 7 & 8.67 \\
\hline$\Sigma^{* 0}-\Sigma^{*+}$ & -4 to 4 [6] & 4.10 & 1.82 & 2.65 & 4 & 6 & 1.96 \\
\hline$\Sigma^{*-}-\Sigma^{* 0}$ & $2.0 \pm 2.46$ & 6.34 & 3.85 & 4.40 & 3 & 4 & 3.69 \\
\hline$\Xi^{-}-\Xi^{0}$ & $6.4 \pm 0.6$ 6] & 10.62 & 7.12 & 9.19 & 3 & 6 & 7.46 \\
\hline$\Xi^{*-}-\Xi^{* 0}$ & $3.2 \pm 0.6$ 6] & 5.87 & 3.68 & 3.58 & 3 & 3 & 3.58 \\
\hline$\Sigma_{c}^{++}-\Sigma_{c}^{0}$ & $0.8 \pm 0.46$ & 0.12 & 1.06 & 2.91 & -2 & 3 & 1.20 \\
\hline$\Sigma_{c}^{+}-\Sigma_{c}^{0}$ & $1.4 \pm 0.6$ 6 & -0.96 & -0.55 & 0.55 & -2 & 1 & -0.36 \\
\hline$\Xi_{c}^{0}-\Xi_{c}^{+}$ & $2.5 \pm 1.7 \pm 1.118$ & 4.67 & 2.58 & 2.90 & 2 & 0 & 2.83 \\
\hline$\Xi_{c}^{\prime 0}-\Xi_{c}^{\prime+}$ & $1.7 \pm 4.618$ & 1.04 & 0.47 & -0.22 & 1 & 0 & 0.30 \\
\hline$\Xi_{c}^{* 0}-\Xi_{c}^{*+}$ & $6.3 \pm 2.6$ 18 & 0.40 & 0.44 & -0.85 & 1 & 0 & 0.43 \\
\hline$\Sigma_{b}^{+}-\Sigma_{b}^{-}$ & & -3.58 & -3.45 & 5.64 & & & -3.57 \\
\hline$\Sigma_{b}^{0}-\Sigma_{b}^{-}$ & & -2.85 & -1.99 & 0.01 & & & -2.51 \\
\hline$\Xi_{b}^{-}-\Xi_{b}^{0}$ & & 7.25 & 5.12 & 4.27 & & & 5.39 \\
\hline
\end{tabular}




\begin{tabular}{|c|c|c|c|c|c|c|c|}
\hline$\Xi_{c c}^{+}-\Xi_{c c}^{++}$ & -1.87 & -2.70 & -5.21 & & & & -2.96 \\
\hline$\Sigma_{c}^{++}-\Sigma_{c}^{+}$ & & 1.08 & 1.61 & 2.36 & 0 & 2 & 1.56 \\
\hline$\Sigma^{+}+\Sigma^{-}-2 \Sigma^{0}$ & & 2.20 & 2.07 & 1.57 & & & 1.81 \\
\hline$\Sigma^{*+}+\Sigma^{*-}-2 \Sigma^{* 0}$ & & 2.24 & 2.03 & 1.75 & & & 1.73 \\
\hline$\Sigma_{c}^{++}+\Sigma_{c}^{0}-2 \Sigma_{c}^{+}$ & & 2.04 & 2.16 & 1.81 & & & 1.92 \\
\hline
\end{tabular}

